\title{
TREE SIGN PATTERN MATRICES THAT REQUIRE ZERO EIGENVALUES
}

\author{
Wei-Hsu Chen and Mao-Ting Chien
}

\begin{abstract}
We characterise tree sign pattern matrices that require at least $k$ zero eigenvalues,
\end{abstract} and exactly $k$ zero eigenvalues.

\section{INTRODUCTION}

A sign pattern matrix $A$ is an $n$-by- $n$ matrix whose entries consist of the symbols ,+- and 0 . Let $Q(A)$ denote the set of all $n$-by- $n$ real matrices which have the same sign as $A$, that is,

$$
Q(A)=\left\{B \in M_{n}(\mathbf{R}): \operatorname{sgn}\left(b_{i j}\right)=a_{i j}, i, j=1,2, \ldots, n\right\}
$$

A sign pattern matrix $A$ is said to require property $P$ if every matrix in $Q(A)$ has property $P$, and allow property $P$ if there exists a matrix in $Q(A)$ which has property $P$. There have been a number of papers $[3,4,5,6,7]$ on sign pattern matrices. In mathematical economics, Quirk and Ruppert [6] studied the stability of sign pattern matrices and characterised sign pattern matrices that require negative real part eigenvalues. Maybee and Quirk [5] introduced graph-theoretic methods to solve qualitative stability of linear systems. Eschenbach and Johnson [2] raised several questions about sign pattern matrices that require or allow certain distributions of eigenvalues. They characterised sign pattern matrices that require all real, all nonreal, and all pure imaginary eigenvalues [3], and also characterised sign patterns that require at least $k$ zero eigenvalues [4]. In [7], Yeh discussed sign pattern matrices that allow a nilpotent matrix.

In this paper, we first decompose the graph of a tree sign pattern matrix into star tree components, and then we characterise tree sign pattern matrices that require at least $k$ zero eigenvalues and exactly $k$ zero eigenvalues in terms of these star tree components.

Received 19th February, 1996

Copyright Clearance Centre, Inc. Serial-fee code: $0004-9729 / 97 \quad \$ A 2.00+0.00$. 


\section{Star Tree Components}

We make use of the terminology and notation that appeared in [1] and [3]. Let $A$ be an $n$-by- $n$ sign pattern matrix. By the graph of $A$, denoted by $G(A)$, we mean the graph with vertex set $\langle n\rangle=\{1,2, \cdots, n\}$, and edge set $E(G(A))$, where an edge $\{i, j\} \in E(G(A))$ whenever $a_{i j} \neq 0 \neq a_{j i}$. A sequence of $l-1$ edges $\left\{i_{1}, i_{2}\right\}$, $\left\{i_{2}, i_{3}\right\}, \cdots,\left\{i_{l-1}, i_{l}\right\}$ in $G(A)$ is called a path of length $l-1$ between $i_{1}$ and $i_{l}$ if $i_{1}, i_{2}, \cdots, i_{l}$ are distinct, and is denoted by $\left\{i_{1}, i_{2}, \cdots, i_{l-1}, i_{l}\right\} ;$ a simple circuit of length $l-1$ between $i_{1}$ and $i_{l}$ if $i_{1}, i_{2}, \cdots, i_{l-1}$ are distinct and $i_{1}=i_{l}$, and is denoted by $\left\{i_{1}, \cdots, i_{l-1}, i_{1}\right\}$. A composite $k$-circuit $r$ is a sequence of simple circuits $r_{1}, r_{2}, \cdots, r_{l}$ whose index sets are mutually disjoint and the sum of whose length is equal to $k$, and is denoted by $r=r_{1} r_{2} \cdots r_{l}$. If $r=\left(i_{1}, i_{2}, \cdots, i_{k}, i_{1}\right)$ is a simple circuit of $G(A)$, its associated circuit product $\gamma=a_{i_{1} i_{2}} a_{i_{2} i_{3}} \cdots a_{i_{k} i_{1}}$ is called a simple cycle of $A$ with length $|\gamma|=k$. A composite k-cycle is a product of simple cycles of a composite $k$-circuit. A simple cycle of $A$ is said to be positive (respectively negative) if it contains an even (respectively odd) number of negative elements in its cycle product. A composite $k$-cycle is positive (respectively negative) if it contains an even (respectively odd) number of negative simple cycles.

An $n$-by- $n$ sign pattern matrix $A=\left(a_{i j}\right)$ is said to be a tree sign pattern (abbreviated as t.s.p.) matrix if (i) $a_{i j} \neq 0$ if and only if $a_{j i} \neq 0 ;(i i) G(A)$ is strongly connected, that is, there is a path between any two vertices; and (iii) there is no simple cycle in $A$ of length 3 or more. The graph $G(A)$ of $A$ is called t.s.p. if $A$ is an t.s.p. matrix. A subgraph $S$ of $G(A)$ is called a star if $S$ contains only simple 2-circuits which share a common vertex. It is clear that $G(A)$ can be expressed as the union of several star subgraphs. For example, $G(A)$ is the union of all one-edge subgraphs of $G(A)$. We are interested in the least number of star subgraphs that compose $G(A)$. Assume that $G(A)$ is the union of star subgraphs $S_{1}, S_{2}, \ldots, S_{k}$ with $E\left(S_{i}\right) \cap E\left(S_{j}\right)=\emptyset$, for $i \neq j$, and where $k$ is minimal. The $k$ star subgraphs are called the star tree components of $G(A)$, and the minimal number $k$ is denoted by $|G(A)|$. The set of all graphs which have $k$ star tree components is denoted by $G_{k}$. It is easy to verify that $G_{1}$ consists of all stars on $n$ vertices, $G_{k}$ is empty if and only if $n<2 k$, and $G_{k}$ consists of paths on $1,2, \ldots, n$ when $k=[n / 2]$. Two star tree components of $G(A)$ are adjacent if they share at a common vertex. An edge and a star tree component of $G(A)$ are adjacent if they share at a common vertex. Observe that an $n$-by- $n$ t.s.p. matrix has simple cycles of length 1 , or 2 . In this paper, we assume that all t.s.p. matrices are nontrivial and have no simple cycles of length 1 , that is, the graph $G(A)$ is loopless.

The following theorem asserts the existence of a star tree component which is located at one end of star tree components decomposition.

Theorem 2.1. Let $A$ be a t.s.p. matrix. Then there exists a star tree compo- 
nent of $G(A)$ which is adjacent to star tree components at only one vertex.

Proof: If the theorem is false then there exists a circuit whose length is more than 2, which contradicts $G(A)$ being a t.s.p. graph.

Let $A$ be a t.s.p. matrix such that $G(A) \in G_{k}$. Any simple 2-circuit in a composite $2 k$-circuit of $G(A)$ is called a component circuit.

The following theorem shows the existence of an $2 k$-circuit of $G(A) \in G_{k}$.

ThEOREM 2.2. Let $A$ be a t.s.p. matrix such that $G(A) \in G_{k}$. Then there exists an $2 k$-circuit of $G(A)$.

Proof: We prove the theorem by induction on $k$. The theorem obviously holds for $k=1$. Assume that the theorem is true for $k \leqslant m$. If $k=m+1$, by Theorem 2.1, there exists a star tree component $S$ in $G(A)$ which is adjacent to star tree components at only one vertex $v$. Suppose there is an 2-circuit $r$ of $S$ which doesn't contain $v$. By the induction assumption, we can find a composite $2 m$-circuit from the remaining $m$ star tree components, and the $2 m$-circuit and $r$ form an $2(m+1)$-circuit. Suppose that each 2-circuit in $S$ contains $v$. Let $r_{1}, r_{2}, \ldots, r_{l}$ be the simple 2-circuits which are adjacent to $S$. Let $\mathcal{H}$ be the union of star tree components of $G(A)$ by removing $S$ and the edges associated with $r_{1}, r_{2}, \ldots, r_{l}$. We claim that there exists an $2 m$-circuit in $\mathcal{H}$. Suppose it is false. Since $\mathcal{H}$ consists of $m$ star subgraphs, it follows that the $m$ star subgraphs are not star tree components for $\mathcal{H}$. Otherwise by the induction hypothesis, there would exist a $2 m$-circuit. Therefore $\mathcal{H}$ can be decomposed into the union of $p$ star tree components with $p<m$. But then the $p$ star tree components and the star subgraph $S \cup\left\{r_{1}, r_{2}, \cdots, r_{l}\right\}$ constitute $p+1$ star tree components for $G(A)$, a contradiction to $G(A) \in G_{m+1}$. Hence there exists a $2 m$-circuit in $\mathcal{H}$, and the $2 m$-circuit and any 2-circuit of $S$ form an 2(m+1)-circuit of $G(A)$.

The following theorem shows that component circuits in a star tree component can be combined with a common $(2 k-2)$-circuit to form $2 k$-circuits.

TheOREM 2.3. Let $A$ be an $n$-by-n t.s.p. matrix such that $G(A) \in G_{k}, k>1$. Suppose $S$ is a star tree component of $G(A)$ and $r_{1}, r_{2}, \cdots, r_{m}$ are component circuits of $S$. Then $\bigcap_{i=1}^{m} \Gamma\left(r_{i}\right) \neq \emptyset$, where $\Gamma\left(r_{i}\right)$ is the collection of all $(2 k-2)$-circuits which combine with $r_{i}$ to form $2 k$-circuits.

Proof: Assume $r_{1}=\left\{a, b_{1}, a\right\}, r_{2}=\left\{a, b_{2}, a\right\}, \cdots, r_{m}=\left\{a, b_{m}, a\right\}$. For each $i=$ $1,2, \cdots, m$, let $T\left(r_{i}\right)$ be the union of star tree components $S_{i_{1}}, S_{i_{2}}, \cdots, S_{i_{j}}$, which are adjacent to $r_{i}$ at $b_{i}$, and the star tree components that are adjacent to $S_{i_{1}}, S_{i_{2}}, \cdots, S_{i_{j}}$. The number of star tree components in $T\left(r_{i}\right)$ is denoted by $\left|T\left(r_{i}\right)\right|$. Suppose that there are $l$ remaining star tree components of $G(A)$ which are not in $\bigcup_{i=1}^{m} T\left(r_{i}\right)$. Since $r_{i}$ is a component circuit of $S$, by Theorem 2.2 , we can find a $2\left|T\left(r_{i}\right)\right|$-circuit $C_{i}$ in $T\left(r_{i}\right)$ 
which is not adjacent to $r_{i}$. Hence the composite circuit $C_{1} C_{2} \ldots C_{m}$ and an $2 l$-circuit in the remaining $l$ star tree components form an $(2 k-2)$-circuit. This composite circuit together with any component circuit $r_{i}, i=1,2, \ldots, m$, form an $2 k$-circuit.

Let $A$ be a t.s.p. matrix. The decomposition of $G(A)$ into the star tree components is not unique. However, if two component circuits lie in a star tree component, they will lie in the same star tree component of any other decomposition.

Theorem 2.4. Let $A$ be a t.s.p. matrix such that $G(A) \in G_{k}$. If $r_{1}, r_{2}$ are two component circuits in a star tree component of $G(A)$, then $r_{1}$ and $r_{2}$ lie in the same star tree component of any other star tree components decomposition of $G(A)$.

Proof: Clearly, the theorem holds for $k=1$. Suppose $k>1$. Suppose $G(A)$ can be decomposed into $k$ star tree components such that $r_{1}$ and $r_{2}$ are contained in star tree components $S_{1}$ and $S_{2}$ respectively. Let $\Gamma\left(r_{i}\right)$ be the collection of all $(2 k-2)$ circuits such that each $(2 k-2)$-circuit and $r_{i}$ form an $2 k$-circuit, $i=1,2$. For every composite circuit $w \in \Gamma\left(r_{1}\right)$, we know that there has to exist an 2-circuit $r$ in $S_{2}$ such that $w$ contains $r$. On the other hand, for any composite circuit $z \in \Gamma\left(r_{2}\right), z$ can't contain $r$ since $r$ and $r_{2}$ are adjacent. Hence $\Gamma\left(r_{1}\right) \cap \Gamma\left(r_{2}\right)=\emptyset$, a contradiction to Theorem 2.3.

\section{Zero Eigenvalues of Tree Sign Pattern Matrices}

Let $B$ be an $n$-by- $n$ real matrix. The sum of all $j$-by- $j$ principal minors of $B$ is denoted by $E_{j}(B)$. It is well-known, see, for example [1, pp.291-292], that $E_{j}(B)$ is the sum of all possible terms of the form

$$
(-1)^{\left|\gamma_{1}\right|-1} \gamma_{1}(-1)^{\left|\gamma_{2}\right|-1} \gamma_{2} \ldots(-1)^{\left|\gamma_{p}\right|-1} \gamma_{p}
$$

where $\gamma_{1}, \gamma_{2}, \ldots, \gamma_{p}$ are disjoint simple cycles of $B$, and the sum of whose length is equal to $j$. The computation of the characteristic polynomial of $B$

$$
P_{B}(t)=t^{n}+\sum_{j=1}^{n}(-1)^{j} E_{j}(B) t^{n-j}
$$

is then expressed in terms of its cycle products.

Let $A$ be an $n$-by- $n$ sign pattern matrix and $\gamma$ be a simple $k$-cycle of $A$. Eschenbach and Johnson [3] introduced two auxiliary matrices for testing the distribution of eigenvalues of sign pattern matrices. One is the matrix $B_{\gamma}(0)=\left(b_{\gamma}(0)\right)_{i j}$ which is defined by

$$
\left(b_{\gamma}(0)\right)_{i j}= \begin{cases}1, & \text { if } a_{i j}=+ \text { and is in } \gamma \\ -1, & \text { if } a_{i j}=- \text { and is in } \gamma \\ 0, & \text { otherwise. }\end{cases}
$$


Another matrix $B_{\gamma}(\varepsilon)=\left(b_{\gamma}(\varepsilon)\right)_{i j} \in Q(A)$, is defined by

$$
\left(b_{\gamma}(\varepsilon)\right)_{i j}= \begin{cases}\left(b_{\gamma}(0)\right)_{i j}, & \text { if } a_{i j} \text { is in } \gamma \\ \varepsilon, & \text { if } a_{i j}=+ \text { and is not in } \gamma ; \\ -\varepsilon, & \text { if } a_{i j}=- \text { and is not in } \gamma ; \\ 0, & \text { otherwise, }\end{cases}
$$

where $\varepsilon$ is a small positive number. By the fact that the eigenvalues depend continously upon the entries of a real matrix, the eigenvalues of the perturbed matrix $B_{\gamma}(\varepsilon)$ are close to that of $B_{\gamma}(0)$. Furthermore, if $\gamma=\gamma_{1} \gamma_{2} \ldots \gamma_{j}$ is a composite cycle, we define $B_{\gamma}(0)$ and $B_{\gamma}(\varepsilon)$ in the same way as that of (1) and (2) respectively.

Let $A$ be an $n$-by- $n$ t.s.p. matrix. In this section, we characterise a t.s.p. matrix that requires at least $k$ zero eigenvalues, and exactly $k$ zero eigenvalues.

TheOREM 3.1. Let $A$ be an $n$-by- $n$ t.s.p. matrix, and $B \in Q(A)$. Then $B$ has an even number of zero eigenvalues if and only if $n$ is even.

Proof: Observe that the characteristic polynomial of $B$ is

$$
P_{B}(t)=t^{n}+\sum_{j=1}^{n}(-1)^{j} E_{j}(B) t^{n-j}
$$

Since $A$ has no cycles of length 1 , the length of each cycle of $A$ is even, and thus $E_{i}(B)=0$ for odd indices. Hence

$$
P_{B}(t)= \begin{cases}t^{n}+\sum_{j=1}^{n / 2} E_{2 j}(B) t^{n-2 j}, & \text { if } n \text { is even } \\ t^{n}+\sum_{j=1}^{(n-1) / 2} E_{2 j}(B) t^{n-2 j}, & \text { if } n \text { is odd }\end{cases}
$$

As a consequence of Theorem 3.1, we have the following result.

THEOREM 3.2. Let $A$ be an $n$-by- $n$ t.s.p. matrix. If $A$ requires exactly $k$ zero eigenvalues then $k$ is even if and only if $n$ is even.

In the following, we characterise an $n$-by- $n$ t.s.p. matrix that requires at least $k$ zero eigenvalues. From Theorem 3.1, we consider only the case when both $n$ and $k$ are even or odd.

TheOREM 3.3. Let $A$ be an $n$-by-n t.s.p. matrix. If $n$ and $k$ have the same parity then $A$ requires at least $k$ zero eigenvalues if and only if the number of star tree components of $G(A)$ is at most $(n-k) / 2$.

PROOF: If $n$ and $k$ are even and the number of star tree components of $G(A)$ is not greater then $(n-k) / 2$, then the largest length of composite circuits of $G(A)$ is less 
than or equal to $n-k$. Hence, for all $B \in Q(A), E_{n-k+2}(B)=E_{n-k+4}(B)=\ldots=$ $E_{n}(B)=0$. By (3), $B$ has at least $k$ zero eigenvalues. Conversely, if the number of star tree components of $G(A)$ is greater than $(n-k) / 2$ then $G(A)$ has a composite circuit, say $\gamma$, of length $l>n-k$. But then $E_{l}\left(B_{\gamma}(0)\right)=1$, or -1 , and thus $E_{l}\left(B_{\gamma}(\varepsilon)\right) \neq 0$ for some $\varepsilon$. By (3) again, $B_{\gamma}(\varepsilon) \in Q(A)$ has at most $n-l<k$ zero eigenvalues, and this proves $(i)$. The odd case can be proved similarly.

In the following, we characterise an t.s.p. matrix that requires exactly $k$ zero eigenvalues.

Theorem 3.4. Let $A$ be an $n$-by- $n$ t.s.p. matrix. Then $A$ requires exactly $k$ zero eigenvalues if and only if

(i) $n$ and $k$ have the same parity;

(ii) $G(A)$ consists of $(n-k) / 2$ star tree components;

(iii) All component circuits in the same star tree component of $G(A)$ have the same sign.

Proof: Suppose $k$ is even.

Necessity. If $A$ requires $k$ zero eigenvalues then, by Theorem $3.1, n$ is even. Suppose $G(A)$ consists of $l$ star tree components. If $l>(n-k) / 2$ then $k>n-2 l$. Let $\gamma$ be a composite $2 l$-cycle. We compute that

$$
P_{B_{\gamma}(0)}(t)=t^{n}+\sum_{j=1}^{l} E_{2 j}\left(B_{\gamma}(0)\right) t^{n-2 j}=t^{n-2 l}\left(t^{2 l}+\sum_{j=1}^{l} E_{2 j}\left(B_{\gamma}(0)\right) t^{2 l-2 j}\right) .
$$

Since $E_{2 l}\left(B_{\gamma}(0)\right)=1$, or -1 , it follows that $E_{2 l}\left(B_{\gamma}(\varepsilon)\right) \neq 0$ for some $\varepsilon$. Hence $B_{\gamma}(\varepsilon) \in Q(A)$ has at most $n-2 l$ zero eigenvalues, and thus has less than $k$ zero eigenvalues, a contradiction. On the other hand, if $l<(n-k) / 2$ then $k<n-2 l$. By (4), we have that, for any $B \in Q(A), B$ has at least $n-2 l$ zero eigenvalues, and so at least $k$ zero eigenvalues, which leads to a contradiction, and thus (ii) follows.

Let $S$ be a star tree component of $G(A)$, and $r_{1}, r_{2}, \cdots, r_{m}$ be component circuits of $S$. Since $A$ requires $k$ zero eigenvalues, we have $E_{n-k}(B) \neq 0$ for all $B \in Q(A)$. If $k=2$ then $G(A)$ itself is a star tree component. In this case, all simple 2-circuits are component circuits, and have the same sign. If $k>2$, by Theorem 2.3, we set the nonempty $C=\bigcap_{i=1}^{m} \Gamma\left(r_{i}\right)$. Then for $B \in Q(A), E_{n-k}(B)=\sum_{i=1}^{m} \gamma_{i}\left(Y_{i}+X\right)$, where $\gamma_{i}$ is the associated cycle of $r_{i}$, and $X$ is the sum of all composite $(n-k-2)$-cycles, each of which is associated with a composite $(n-k-2)$-circuit from $C$, and the remaining terms in the summands of $E_{n-k}(B)$ are collected by $\gamma_{i} Y_{i}, i=1,2, \cdots, m$. If there exists $i \neq j$ such that $\gamma_{i}\left(Y_{i}+X\right)$ and $\gamma_{j}\left(Y_{j}+X\right)$ have opposite signs, we may adjust the values of $\gamma_{i}$ and $\gamma_{j}$ so that $E_{n-k}(B)=0$. Therefore all of the terms $\gamma_{i}\left(Y_{i}+X\right), i=$ 
$1,2, \cdots, m$ have the same sign. Notice that $Y_{i}$ is the sum of all composite $(n-k-2)$ cycles, and each of its associated composite $(n-k-2)$-circuits is adjacent to $S$. For each such composite $(n-k-2)$-cycle from $Y_{i}$, we place a sufficiently small value on the simple 2-cycle whose associated 2-circuit is adjacent to $S$. Then the value $\gamma_{i}\left(Y_{i}+X\right)$ is approximated by $\gamma_{i} X$, and thus $r_{i}, i=1,2, \cdots, m$, have the same sign, and (iii) is proved.

Sufficiency. If $n$ is even and $G(A)$ consists of $(n-k) / 2$ star tree components, then for all $B \in Q(A), E_{l}(B)=0$ for all $n-k<l \leqslant n$. Furthermore, since all component circuits in the same star tree component have the same sign, it follows that $E_{n-k}(B) \neq 0$ for all $B \in Q(A)$. Hence $A$ requires exactly $k$ zero eigenvalues.

A similar argument applies for odd $k$.

Examples. Consider the sign pattern matrix

$$
A=\left[\begin{array}{ccc}
0 & + & + \\
+ & 0 & 0 \\
- & 0 & 0
\end{array}\right]
$$

Then $G(A) \in G_{1}$, and by $(i i)$ of Theorem 3.3, $A$ requires at least one zero eigenvalue.

On the other hand, consider the sign pattern matrix

$$
A=\left[\begin{array}{ccc}
0 & + & - \\
+ & 0 & 0 \\
- & 0 & 0
\end{array}\right]
$$

Then $G(A) \in G_{1}$, and the component circuits in the only one star tree component of $G(A)$ have the same positive sign. Hence, by Theorem $3.4, A$ requires exactly one zero eigenvalue.

\section{REFERENCES}

[1] R. Brualdi and H.J. Ryser, Combinatorial matrix theory (Cambridge Univ. Press., Cambridge, 1991).

[2] C.A. Eschenbach and C.R. Johnson, 'Several open problems in qualitative matrix theory involving eigenvalue distribution', Linear and Multiliner Algebra 24 (1988), 79-80.

[3] C.A. Eschenbach and C.R. Johnson, 'Sign patterns that require real, nonreal or pure imaginary eigenvalues', Linear and Multilinear Algebra 29 (1991), 299-311.

[4] C.A. Eschenbach and C.R. Johnson, 'Sign patterns that require repeated eigenvalues', Linear Algebra Appl. 190 (1993), 169-179..

[5] J. Maybee and J. Quirk, 'Qualitative problems in matrix theory', SIAM Rev. 11 (1969), 30-51. 
[6] J. Quirk and R. Ruppert, 'Qualitative economics and the stability of equilibrium', Rev. Econom. Stud. 32 (1965), 311-326.

[7] L. Yeh, 'Sign pattern matrices that allow a nilpotent matrix', Bull. Austr. Math. Soc. 53 (1996), 189-196.

Department of Mathematics

Soochow University

Taipei

Taiwan 11102 